\title{
TAIEX INDEX OPTION MODEL BY USING NONLINEAR DIFFERENTIAL EQUATION
}

\author{
Tsung-Jui Chiang Lin ${ }^{1}$, Meng-Rong $\mathrm{Li}^{2}$ and Yong Shiuan Lee ${ }^{3}$ \\ ${ }^{1}$ Graduate Institute of Finance, National Taiwan University of Science and \\ Technology, 10607, Taipei, Taiwan \\ ${ }^{2}$ Department of Mathematical Sciences, National Chengchi University, 11605 \\ Taipei, Taiwan \\ ${ }^{3}$ Department of Statistics, National Chengchi University, 11605, Taipei, Taiwan \\ D9918005@mail.ntust.edu.tw, hierteli@gmail.com,99354501@ nccu.edu.tw
}

\begin{abstract}
In this study we treat TXO price as a dynamic system which changes over time and characterize it by differential equations. Our goal is to construct a model more suitable for TXO. We use "Parabola Approximation" proposed by Li et al. (2011) to solve the differential equations and try to find the model which fits our data the most. Empirical study shows the model used all produce accurate estimates of TXO prices.
\end{abstract}

Key Words- Differential Equation, Dynamic System, Parabola Approximation, Options, TXO, B-S Model

\section{INTRODUCTION}

Main types of derivatives are forward contracts, futures, options and swaps. Option is an agreement that holders can trade the underlying asset at some time in the future (the expiration date or maturity) for a certain price (the exercise price or strike price). Holders must pay the premium to sellers who are obligated to carry out the contract at the expiration date. If it is possible to forecast the theoretical price of an option by using a good model, the participants in the market could have better control of cost or understand if there exists arbitrage opportunity. For a country or a section, the good theoretical price forecast also provides stability of the option trading market.

Taiwan Futures Exchange provides a variety of derivatives as Taiwan financial market flourished. Among them, the trading volume of TAIEX index option (TXO) increases the most since traded in 2001 and now is the largest. Since TXO is popular in Taiwan financial market, building a reliable and accurate model to explain the change of its price or forecast its theoretical price is instant and also a main research subject in Taiwan.

In this study, we regard the market prices of some TXO contracts as a dynamic system which is a continuous function of time respectively. From real data, we try to find the relationship between the option price and time as characterizing it by differential equations. By this approach we expect to build a model which fits Taiwan empirical data the most and offer a new method for financial research. This article is divided into five main sections. Section 1 provides a brief introduction to the motive and purpose of the study and reviews important literature and models. Section 2 outlines the analysis method used in this study. Section 3 presents the comparison of empirical study results of our model and Black-Scholes model. Finally, section 4 provides conclusions and suggestions for future studies. 
Literature There are many option pricing models, among which the most popular is Black-Scholes (B-S) model [1] and other improved models based on it. In B$\mathrm{S}$ model, the stock price is viewed as a stochastic process and therefore the option pricing formula is derived. Under certain boundary conditions, there exists the unique solution of B-S and is very convenient when being applied to empirical data; however the derivation is complicated and it is difficult to explain the model coefficients economically. This makes lots of researchers try to improve B-S model in order to produce theoretical values approaching market values, for example in [2 4].

Since B-S model can only be applied to value European options, CRR model [5] is used to value American options, which is a discrete-time model. As the time interval becomes smaller, the theoretical option price calculated from this model will converge to that of continuous-time model in the limit. Hence this model can be seen as a special case of B-S model. As for valuing other options, details and discussion can be found in Options, Futures, and Other Derivatives [6].

Based on previous results of empirical studies, we simply assume the price of stock and the price of option are continuous functions of time and can be regarded as a dynamic system respectively. We study the change of the dynamic system over time and construct the model of it from real data without specifying the form of model at first. We then recognize the economic meanings of the coefficients in the model. By this approach, we try to form a model more applicable to reality and the theoretical values closer to the market values.

\section{METHOD}

The evolution rule of a dynamic system is generally represented by a differential equation which sometimes is a partial differential equation because the state space could have many dimensions. Furthermore, in reality the equation is frequently nonlinear since the phenomenon exists in the fields such as fluid mechanics, automatic control, etc. As a result, the numerical computation of nonlinear differential equation and dynamic system trace back to fluid mechanics [12,13], and afterwards it was applied to biology, ecology, Logistic equation in population dynamics, electronic circuit, and artificial intelligence and so on.

We treat our subject $u(t)$ as a dynamic system whose evolution rule can be represented by a nonlinear differential equation: $d u / d t=f(t, u)$. Generally the initial condition is given as $u(0)=u_{0}$.

In real world it is hardly to know the exact form of $f(t, u)$, and hence in this study we use parabola approximation proposed by $\mathrm{Li}$ [10] to obtain better approximate solution. We expand the function $f(t, u)$ at the given initial point $t=t_{0}$ and $u=u_{0}$ by Taylor's Theory as $\left.f(t, u) \sim \sum_{n=0}^{\infty} \frac{1}{n !}\left(\frac{\partial}{\partial t} t+\frac{\partial}{\partial u} u\right)^{n} f\right|_{t=t_{0}, u=u_{0}}(t, u)$, where $\left.\left(\frac{\partial}{\partial t} t+\frac{\partial}{\partial u} u\right)^{n} f\right|_{t=t_{0}, u=u_{0}}(t, u)$ is the binomial expansion at the given initial point $t=t_{0}$ and $u=u_{0}$ : $\left.\sum_{k=0}^{n} \frac{\partial^{n} f(t, u)}{\partial t^{k} \partial u^{n-k}}\right|_{t=t_{0}, u=u_{0}}\left(t-t_{0}\right)^{k}\left(u-u_{0}\right)^{n-k}$. Therefore our question $d O(t) / d t=f(t, O)$ can be approximated to: 


$$
\frac{d O(t)}{d t}=\alpha(t)\left(O(t)-O_{0}\right)^{2}+\beta(t)\left(O(t)-O_{0}\right)+\gamma(t), O\left(t_{0}\right)=O_{0},
$$

where $\quad \alpha(t)=f_{O O}\left(t_{0}, O_{0}\right) / 2, \quad \beta(t)=f_{t, O}\left(t_{0}, O_{0}\right)\left(t-t_{0}\right)+f_{O}\left(t_{0}, O_{0}\right), \gamma(t)=f_{t t}\left(t_{0}, O_{0}\right)\left(t-t_{0}\right)^{2} / 2+$ $f_{t}\left(t_{0}, O_{0}\right)\left(t-t_{0}\right)+f\left(t_{0}, O_{0}\right)$. If the differential equation satisfies

$$
\left\{\begin{array}{l}
\frac{d O(t)}{d t}=\alpha O(t)^{2}+\beta O(t)+\gamma, \\
O\left(t_{0}\right)=O_{0}, O\left(t_{1}\right)=O_{1}, O\left(t_{2}\right)=O_{2}, t_{0} \leq t_{1} \leq t_{2}
\end{array},\right.
$$

we calculate the difference of the data as the observation of $d O(t) / d t$ and then the coefficients $\alpha, \beta, \gamma$ in (2) every three successive data. In this manner we obtain the theoretical value of $O(t)$ by parabola approximation piecewisely. Since the order of observations are not necessarily consistent with time, the sign of integral needs to be negative when they differ. We discuss 6 different situations during the process of solving the differential equation by parabola approximation as follows:

$$
O_{0} \leq O_{1} \leq O_{2}, O_{0} \leq O_{2} \leq O_{1}, O_{1} \leq O_{0} \leq O_{2}, O_{1} \leq O_{2} \leq O_{0}, O_{2} \leq O_{0} \leq O_{1}, O_{2} \leq O_{1} \leq O_{0} .
$$

Also, the discriminant of the equation, $\delta=\beta^{2}-4 \alpha \gamma$, may be greater than, equal to, or less than zero. If the discriminant is greater than zero, we denote the two roots of the equation as $x^{1}$ and $x^{2}$, where $x^{1} \leq x^{2}$. Then we can categorize the solutions by the order of observations and the discriminant.

As solving the differential equation by parabola approximation, sometimes the coefficient $\alpha$ is very close to zero. Under the circumstances, we should solve the differential equation by both linear and parabola approximation. Since the integral is closer to real data and error reduces when solving the differential equation by linear approximation if successive data go straight up or down.

Additionally, if the two roots of the equation as $x^{1}$ and $x^{2}$ fall in the range of integration (i.e. at least one value of $x^{1}$ and $x^{2}$ is between $O\left(t_{0}\right)$ and $O(t)$ ), the integral is unreliable because their singularity properties. We also solve the differential equation by linear approximation in such situation.

Application to options Suppose our research subject is denoted as $u(t)$, whose evolution rule is characterized by a nonlinear differential equation and solved by parabola approximation described previously. In this study our subject is TXO (There are two types: Call and Put. In this study we take call option as example to explore the effect of constructing model by nonlinear differential equation and dynamic system.) or Taiwan stock index. We make some notations related to our subject $u(t)$ :

$S(t)$ : Average price of Taiwan stock index at time $t$

$C(t)$ : Average price of TXO call option at time $t$

$v_{S}(t):=d S(t) / d t$, changing rate of stock index at time $t$

$v(t):=d C(t) / d t$, changing rate of average call price at time $t$

We use forward difference method in this study, i.e. we take $C(t+1)-C(t)$ as the differential of $C(t), d C(t) / d t$, and $S(t+1)-S(t)$ as the differential of $S(t), d S(t) / d t$. We compare two methods - forward and backward difference - and the empirical analysis results agree. For the convenience of representing the model we only show the result of forward difference method below. 
We assume both average call price and stock index are functions of time. We propose the following models to study their relationship with time:

$$
\left\{\begin{array}{l}
\frac{d O(t)}{d t}=\alpha O(t)^{2}+\beta O(t)+\gamma \\
O\left(t_{0}\right)=O_{0}, O\left(t_{1}\right)=O_{1}, O\left(t_{2}\right)=O_{2}, t_{0} \leq t_{1} \leq t_{2},
\end{array}\right.
$$

where $\alpha$ represents the relationship of level of risk per dollar and average call price, $\beta$ means the level of risk and $\gamma$ is the growth rate of average call price relevant to economic scale and market information by time $t$.

As using this model to fit the data, we separate the data into pieces among which each contains three successive data and then find the relationship between the subject (average call price or stock index) and its changing rate by parabola approximation piecewisely. First we solve the differential equation

$$
v(t)=\alpha_{j} C(t)^{2}+\beta_{j} C(t)+\gamma_{j}, C(t) \in I_{j}, \text { or } v_{S}(t)=\alpha_{j} S(t)^{2}+\beta_{j} S(t)+\gamma_{j}, S(t) \in I_{j} .
$$

Afterwards integrating the equation above over the time interval (i.e. applying the results in last section) allows us to obtain the theoretical values of average call price or stock index. When we apply this model to average call price and solve the differential equations by parabola approximation, calculations and meanings of the coefficients $\alpha$, $\beta, \gamma$ are in the following. As using this model to fit the data, from differential equation (3) we obtain $v_{0}=\alpha C_{0}^{2}+\beta C_{0}+\gamma, v_{1}=\alpha C_{1}^{2}+\beta C_{1}+\gamma, v_{2}=\alpha C_{2}^{2}+\beta C_{2}+\gamma$. Then we can derive

$$
\alpha=\left(\frac{v_{2}-v_{1}}{C_{2}-C_{1}}-\frac{v_{1}-v_{0}}{C_{1}-C_{0}}\right) /\left(C_{2}-C_{1}\right) \text {. }
$$

If we define $m_{0}{ }^{\prime}=\left(v_{1}-v_{0}\right) /\left(C_{1}-C_{0}\right)$ as the slope of changing rate of average call price $v(t)$ and average call price $C(t)$ between time 0 and $1, m_{1}{ }^{\prime}=\left(v_{2}-v_{1}\right) /\left(C_{2}-C_{1}\right)$ as the slope of changing rate of average call price $v(t)$ and average call price $C(t)$ between time 1 and 2, we can derive the formulae of $\alpha, \beta, \gamma$ respectively as

$$
\alpha=\frac{m_{1}{ }^{\prime}-m_{0}{ }^{\prime}}{C_{2}-C_{1}}, \beta=\frac{1}{C_{2}-C_{1}}\left(m_{0}{ }^{\prime} C_{2}\right)+\frac{C_{0}}{C_{2}-C_{1}}\left(m_{0}{ }^{\prime}-m_{1}{ }^{\prime}\right), \gamma=v_{0}-\frac{C_{0}}{C_{2}-C_{1}}\left(m_{0}{ }^{\prime} C_{2}-m_{1}{ }^{\prime} C_{1}\right) .
$$

In this study we use two kinds of errors to evaluate the estimation effects of the models. Definitions of these two errors are Mean Absolute Percentage Error (MAPE):

$$
\frac{1}{n} \sum_{i=1}^{n}\left|\left(\hat{O}_{i}(t)-O_{i}(t)\right) / O_{i}(t)\right| \times 100 \%
$$

and Root Mean Square Percentage Error (RMSPE):

$$
\frac{1}{n} \sqrt{\sum_{i=1}^{n}\left(\left(\hat{O}_{i}(t)-O_{i}(t)\right) / O_{i}(t)\right)^{2}} \times 100 \%,
$$

where $\hat{O}_{i}$ is the theoretical value of the model at time $i, O_{i}$ is the real value of the data at time $i$, and $n$ is the number of data. The smaller MAPE and RMSPE are, the better the model fits. The results of using them to evaluate models are similar in this study. MAPE is a measure of accuracy of how well the model fits the data. We use the classification suggested by Lewis [7]:

$$
\begin{array}{ccccc}
\multicolumn{5}{c}{\text { Table 1 }} \\
\text { MAPE } & <10 \% & 10 \% \sim 20 \% & 20 \% \sim 50 \% & >50 \% \\
\text { Accuracy } & \text { High } & \text { Good } & \text { Reasonable } & \text { Inaccurate }
\end{array}
$$




\section{EMPIRICAL STUDY}

\subsection{Data collection and processing}

Historical trading data of TXO is provided by Taiwan Futures Exchange (TAIFEX) and the data are recorded per second. Historical trading data of Taiwan stock index (TAIEX) is provided by Taiwan Stock Exchange (TWSE) and the data are recorded per minute. In this study we randomly select TXO call options with one-month duration from 2008 trading data. The TXO call options selected are traded from Sep. 18, 2008 to Oct. 15, 2008. Based on the closing price of Taiwan stock index on Sep. 17, 2008 (5800.87), we select 5 TXO call options as subjects to study their market prices during the one-month duration. The 5 TXO call options have strike prices as 5600 (inthe-money), 5700 (in-the-money), 5800 (at-the-money), 5900 (out-of-the-money), and 6000 (out-of-the-money) respectively.

Historical trading data of TXO show that trades spread unevenly, that is, there may be several trades in one minute and there may not be any trade during a period of time. On the other hand, the trading time of stock in Taiwan is from 9:00 AM to 1:30 PM while the trading time of TXO is from 8:45 AM to 1:45 PM. Therefore, we calculate the average price of a call from 8:45 AM to 9:00 AM and average price from 1:30 PM to 1:45 PM to be correspondent with the trading time of stock. For the convenience of calculations of differentials in the nonlinear differential equation, we then process the data to obtain average price of each TXO call option and stock index per 90 minutes. As a result, the number of data is 54 for the average stock index series and also 54 for each series of average call price.

\subsection{Model fitting}

We assume both average price of TXO call option and stock index are related to time. Then we fit the data by dynamic model stated previously and compare the differences between fitted values and real values. The results of model fits are similar for all five average call price series, so we take the average price of the at-the-money call (i.e. strike price is 5800) and stock index for example as follows to illustrate the fits of the dynamic model.

As using dynamic model to fit the data, we integrate directly to obtain the theoretical values of average call price or stock index. Figure 1 and Figure $2^{1}$ show the comparison results of model theoretical values and market values as follows. From both figures, the variation tendency of theoretical values is similar to that of market values in spite of lags of theoretical values at some turning points. MAPEs of model for fitting average price of the at-the-money call and stock index are $17.40 \%$ and $0.45 \%$ respectively. The model fitting is good, especially highly accurate for stock index.

\footnotetext{
${ }^{1}$ When $t \in[46,48]$, we use linear approximation to calculate the theoretical value at the $48^{\text {th }}$ position as introduced in the research method section. However the slope between the $48^{\text {th }}$ and the $49^{\text {th }}$ points are too large (8.95), the theoretical value is overestimated. As a result, we use $d S / d t(47)=28.9, S^{*}(48)=S(47)+d s / d t$ to calculate the theoretical value, where $S^{*}(t)$ is the theoretical value of stock index at time $t$.
} 


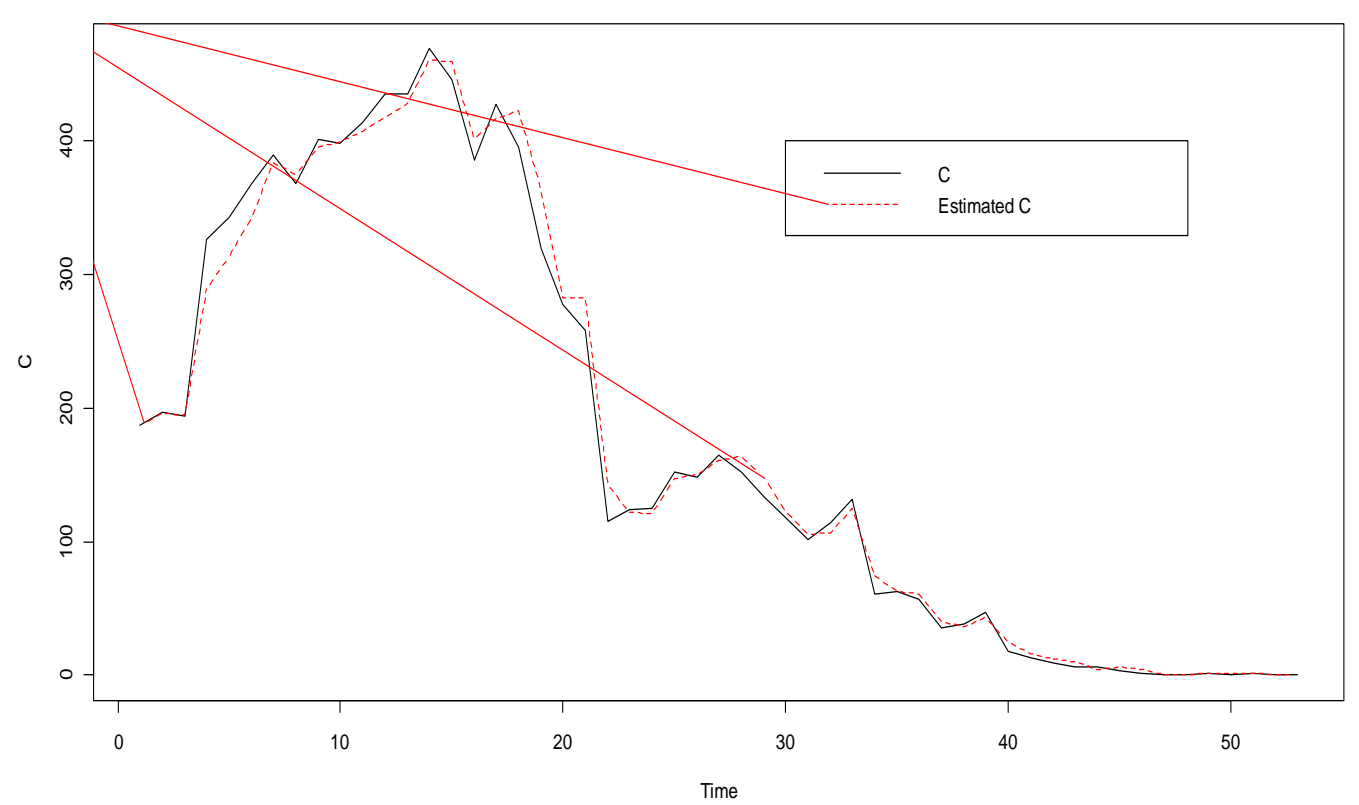

Figure 1. Result of average call price (exercise price 5800)

To understand the characteristics of dynamic model better, we analyze the same data by B-S model to compare the performances of these two sorts of models. The variables we need during the process of valuing options by B-S model are in the following. Stock index and strike price are known. We take one-month interest rates on deposits of September and October, 2008, announced by Bank Of Taiwan to be the risk-free interest rates. The time to maturity is calculated by the proportion of actual trading days to maturity to total trading days in the year. Volatility is calculated from the same data of stock index we use by Historical volatility model (variance-covariance model). 


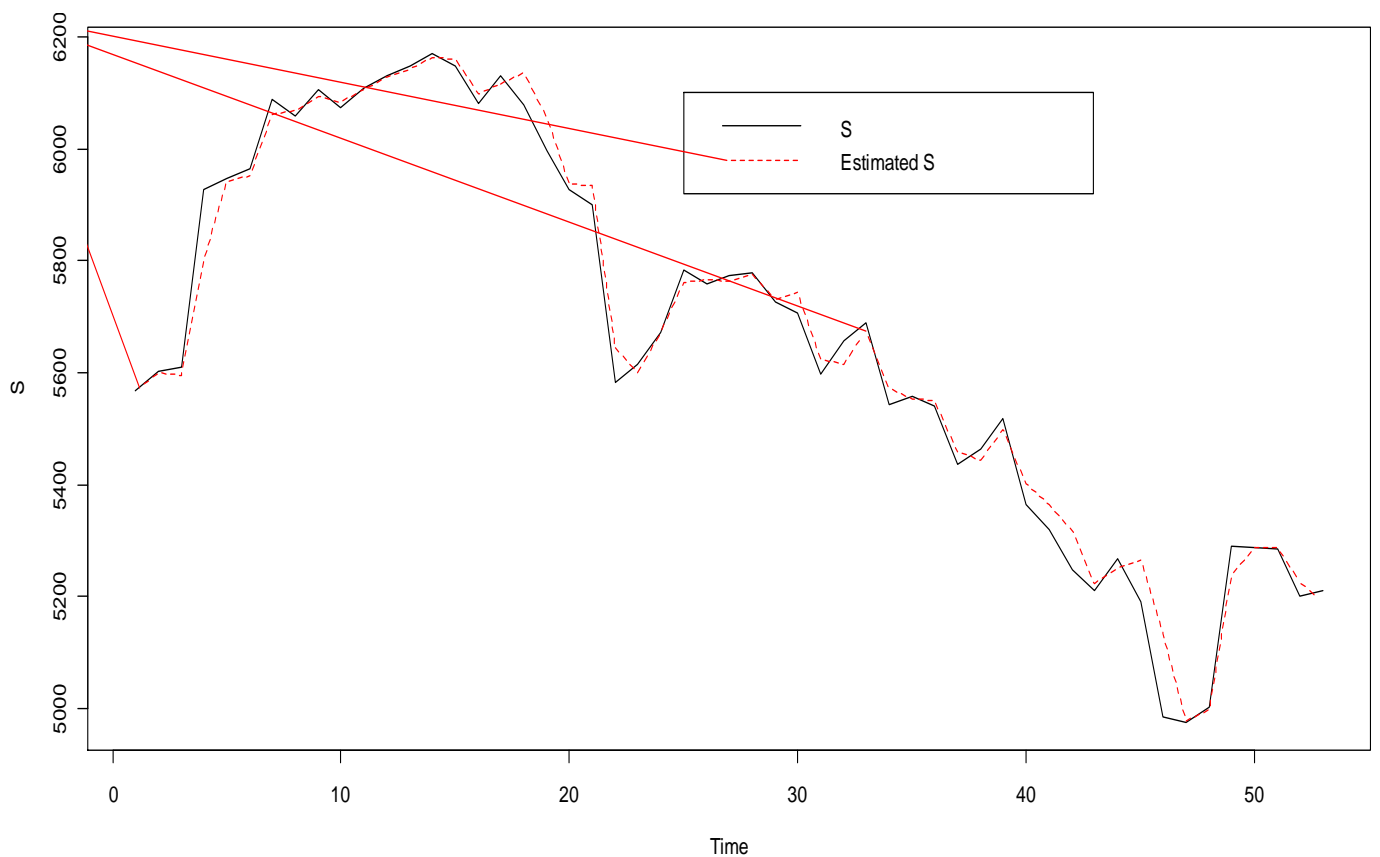

Figure 2. Result of stock index

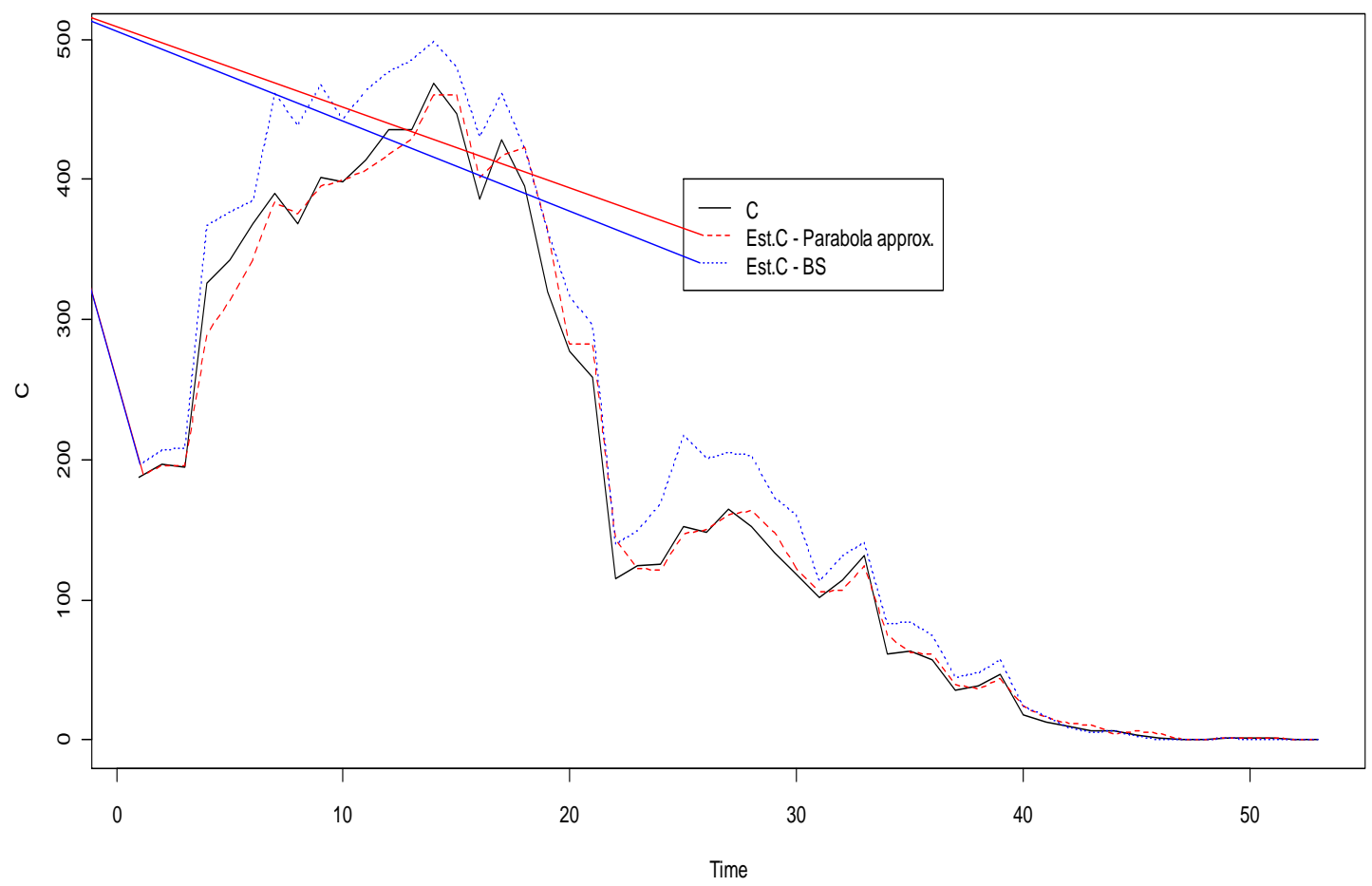

Figure 3. Comparisons between dynamic and B-S model - average price of call (exercise price 5800)

We take the average price of the at-the-money call for example, comparisons of the theoretical values of the dynamic model, the theoretical values of B-S model, and market prices are shown in Figure 3 As mentioned before, the theoretical values of 
dynamic model are very close to market prices while B-S model can capture the variation tendency of the data but with more obvious errors. We consider this as a result of estimation of volatility. Former researches indicate that volatility is the vital variable in valuing options by B-S model and cannot be known in advance or directly observed from market information, it is the most adequate to use Historical volatility model to estimate volatility because it produces theoretical values closer to market values [11]. However the error of B-S model is somewhat larger compared with dynamic model; consequently we need a more precise model in application.

In this study we analyze the data of five average call price series and the comparison results of dynamic model and B-S model is in the following:

Table 2. Table 3.

\begin{tabular}{|c|c|c|c|c|c|}
\hline MAPE & $\begin{array}{c}\text { parabola } \\
\text { model }\end{array}$ & $\begin{array}{c}\text { B-S } \\
\text { model }\end{array}$ & RMSPE & $\begin{array}{c}\text { parabola } \\
\text { model }\end{array}$ & $\begin{array}{c}\text { B-S } \\
\text { model }\end{array}$ \\
\hline Strike price 5600 & $31.14 \%$ & $17.22 \%$ & Strike price 5600 & $13.89 \%$ & $3.32 \%$ \\
\hline Strike price 5700 & $52.95 \%$ & $21.52 \%$ & Strike price 5700 & $24.57 \%$ & $4.16 \%$ \\
\hline Strike price 5800 & $17.40 \%$ & $26.51 \%$ & Strike price 5800 & $5.81 \%$ & $4.94 \%$ \\
\hline Strike price 5900 & $12.44 \%$ & $32.63 \%$ & Strike price 5900 & $3.53 \%$ & $5.74 \%$ \\
\hline Strike price 6000 & $14.62 \%$ & $39.02 \%$ & Strike price 6000 & $3.93 \%$ & $6.53 \%$ \\
\hline Average & $25.71 \%$ & $27.38 \%$ & Average & $10.35 \%$ & $4.94 \%$ \\
\hline
\end{tabular}

Table 2 MAPEs of dynamic model and B-S model - average price of call,

Table 3 RMSPEs of dynamic models and B-S model - average price of call.

This dynamic model can capture the variation tendency of the data as a whole. Although the errors of this model when fitting average prices of out-of-the-money call options are a little larger, the errors of the rest data show that model fittings are good. After examining the fitting results, we discover that the lager errors show when the call options are near the expiration date. In the situation, if the call is out-of-the-money and the value of the call is close to zero, then slight fluctuations in market prices will cause severe fluctuations in nonlinear theoretical values and large errors. B-S model also can capture the variation tendency of the data but with obviously larger errors. Despite of larger errors, the theoretical values of B-S model can converge to zero when the call options are near the expiration date.

\subsection{Graphs of coefficients of model}

When we study our subject, TXO, by applying dynamic model, it is important to find out the functions which characterize the relationship between these coefficients and time. From the functions, we have clues of the factors that may affect average prices of TXO, sources of errors, and predictions of the subject. Empirical study shows that the dynamic model has good fits, we take the average price of the at-the-money call (exercise price 5800) for example, graphs of $\alpha(t), \beta(t), \gamma(t)$ in dynamic model vs. time are drawn in Figure 4, 5 and 6 respectively in the following. 


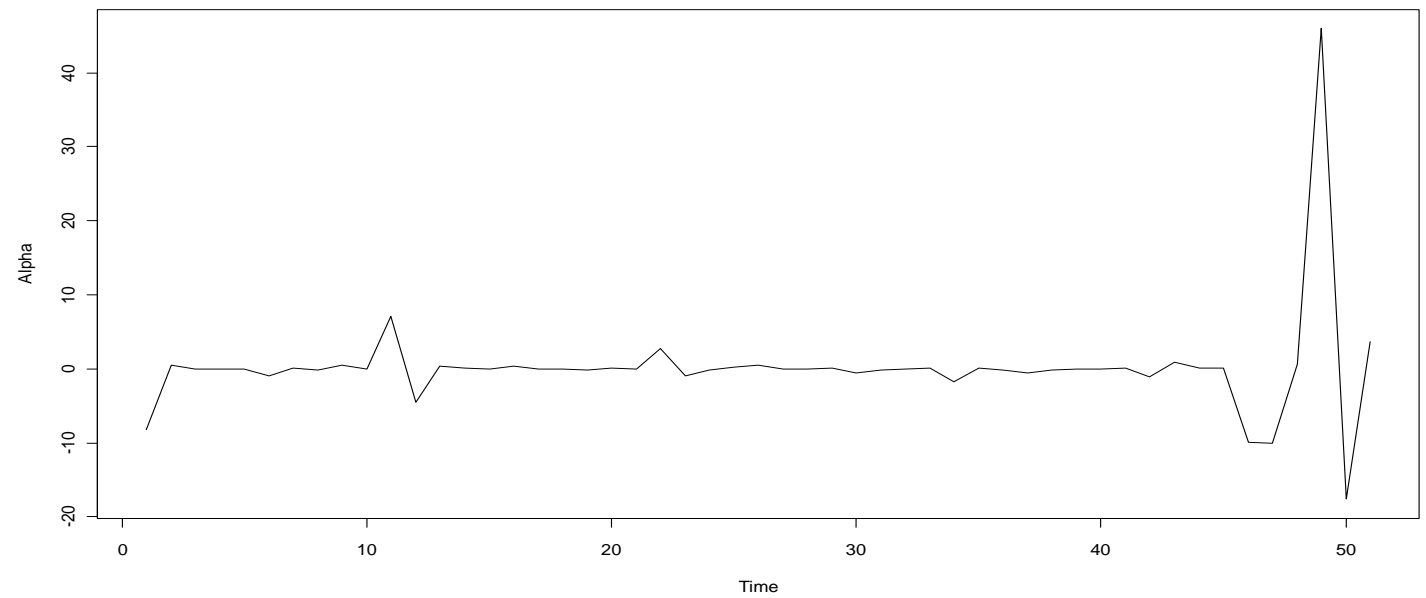

Figure 4. $\alpha(\mathrm{t})$

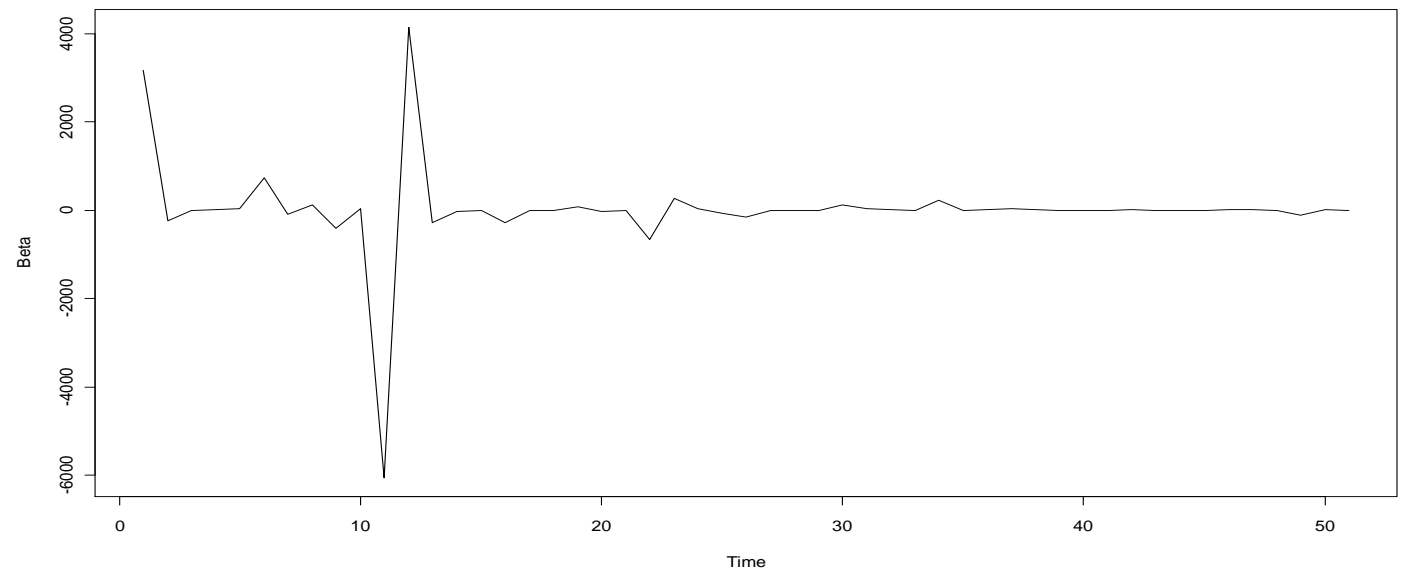

Figure 5. $\beta(\mathrm{t})$

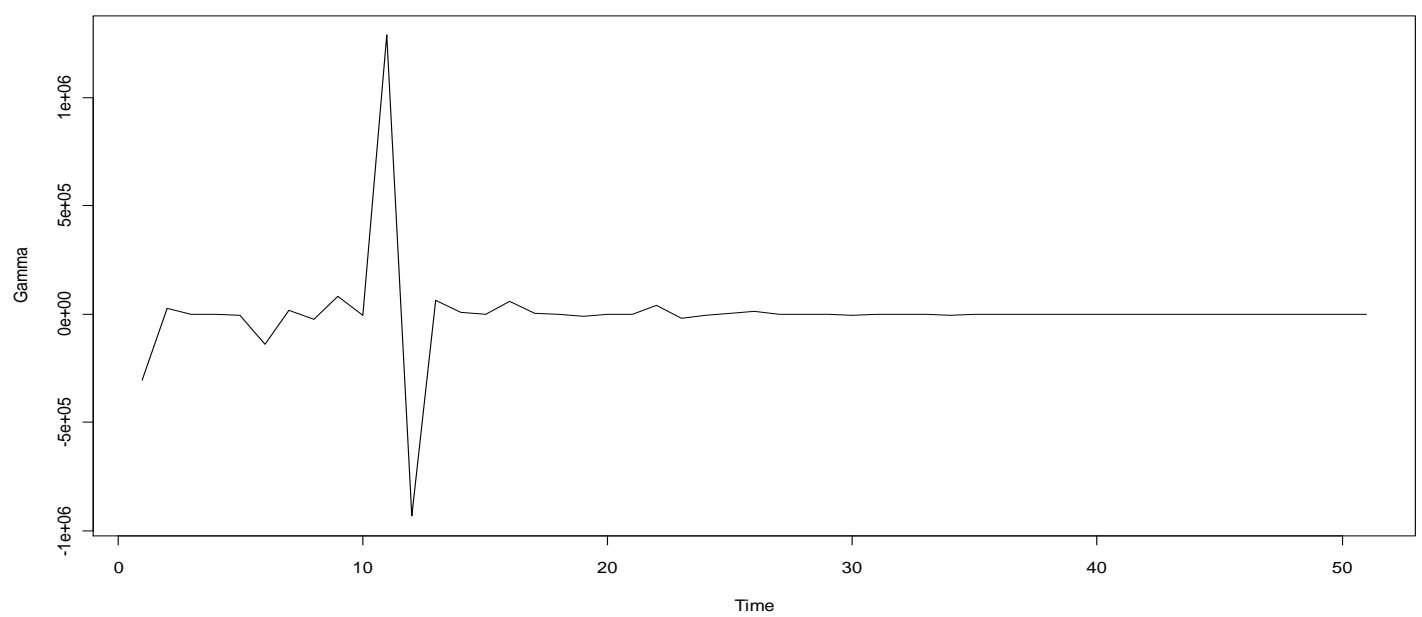

Figure $6 \gamma(\mathrm{t})$ 


\section{CONCLUSIONS AND SUGGESTIONS}

B-S model has been the most important model for valuing options since nineteen seventies, and other improved models are mostly based on the assumptions of B-S model. But B-S model still produces unreasonable errors due to the differences between reality and the model assumptions and all the assumptions cannot be corrected at the same time. Additionally, in order to correct the assumption of constant volatility in B-S model, improved model assumes the variance of return change with fixed elasticity but the assumption of volatility is strong in this way. Furthermore, many researches indicate that estimation of volatility is the most difficult part when applying B-S model and the estimation directly affect the precision of the model. In this study, we consider the options valuation from another point of view. Hence we do not have any assumptions on market and build the model from real data of the subject.

We regard the main subject (TXO or stock index) as a dynamic system which changes over time. We only assume the evolution rule is a continuous function of time and can be represented by a differential equation. Then we use parabola approximation to find the model which fits the data the most. Both dynamic model and B-S model assume stock price change with time, but B-S model assume it follows the specific movement-geometric Brownian motion - while dynamic model does not specify how it moves. Therefore, there exists the unique solution of $\mathrm{B}-\mathrm{S}$ model under certain boundary conditions and it is very convenient when being applied to empirical data. On the other hand, when applying dynamic model we have to find the functions to describe how the coefficients change with time and hence there are more steps of numerical computations.

Empirical study shows that this dynamic model in this paper has good fits for average call prices and even highly accurate fits for stock index, and characterizes actual changes of the subject. We can conclude that both prices of TXO call options and stock index (TAIEX) can be modeled by dynamic system model. If we can predict stock index precisely in the future, we can directly value any derivatives based on the prediction of the underlying stock index. We make some suggestions of future researches as follows:

1. Empirical study shows dynamic model theoretical values of average call prices do not converge to zero as B-S model does. Dynamic model is more sensitive and the theoretical values sometimes fluctuate when the call options are near the expiration date because the call is out-of-the-money and the value of the call is close to zero. Furthermore we should consider the price limits in Taiwan stock market. So we suggest that some constraints may be added to the dynamic model in order to avoid the fluctuations mentioned above.

2. All the coefficients of dynamic model are time-related. From the calculations of their functions, we find them to be very dynamical so that it may cause some difficulties to predict the theoretical values of options prices. Except for predicting the subject from directly predicting the coefficients, we can first model the coefficients by considering the effects of some economic variables (e.g. exchange rate between NTD and USD) and then predict the behavior of the subject. By considering the effects of economic variables, we may understand the causes of the coefficients' dynamics and lower errors of predictions. 
3. In practice, the data of options trades are not collected at the fixed time so that intervals of each trade are not the same. Furthermore, errors occur when applying continuous-time model to discrete data. In order to lower errors, we suggest that interpolate artificial data to shorten intervals between trades without affecting the theoretical values.

In this paper, we study the subject, TXO call options, and investigate the possibility of valuing options by dynamic model. Empirical study shows that we can obtain accurate model fits in this way. According to MAPE, the dynamic model capture the trend and details of changes of the data and produce good fits. In the future, we will try to confirm the sources of errors and economic meanings of dynamic model. Moreover, we will apply this method to value both call and put options with different durations and other derivatives based on the prediction of the underlying stock. Thus, we hope to offer another model to value derivatives.

\section{REFERENCES}

1. F. Black and M. Scholes, The Pricing of Options and Corporate Liabilities, Journal of Political Economy 81(3), 637-654, 1973.

2. T. Bollerslev, Generalized Autoregressive Conditional Heteroskedasticity, Journal of Econometrics 31, pp. 307-327, 1986.

3. P.P. Boyle and T. Vorst, Option Replication in Discrete Time with Transaction Cost, Journal of Finance 47, 271-294, 1992.

4. D.P. Chiras and S. Manaster, The Information Content of Option Prices and a Test of Market Efficiency, Journal of Financial Economics 6, 213-234, 1978.

5. J.C. Cox, S.A. Ross and M. Rubinstein, Option Pricing: A Simplified Approach, Journal of Financial Economics 7, 229-263, 1979.

6. J.C. Hull, Options, Futures, and Other Derivatives, Prentice Hall, New York, 2005.

7. C.D. Lewis, Industrial and Business Forecasting Methods, Butterworths, London, 1982.

8. M.R. Li, Estimates for the Life-Span of the Solutions for Semilinear Wave Equations, Communications on Pure and Applied Analysis 7(2), 417-432, 2008.

9. M.R. Li, On the Blow-up Time and Blow-up Rate of Positive Solutions of Semilinear Wave Equations $\square \mathrm{u}-\mathrm{u}^{\mathrm{p}}=0$ in 1-dimensional Space, to appear on Communications on Pure and Applied Analysis (CPAA), 2008.

10. M.R. Li , T.H. Shieh, C.J. Yue , P. Lee and Y.T. Li, Parabola Method in Ordinary Differential Equation, Taiwanese Journal of Mathematics 15(4), 1841-1857, 2011.

11. Y.S. Ni, M.H. Wu and Y.W. Jeng, The Method of the Volatility Estimator in TXO Under the Black-Scholes model, Management Science Research 2(1), 93-109, 2005.

12. T.H. Shieh and M.R. Li, Numerical Treatment of Contact Discontinuity with Multigases, Journal of Computational and Applied Mathematics 230(2), 656-673, 2009.

13. T.H. Shieh,T.M. Liu, M.R. Li (), Analysis on Numerical Results with Different Exhaust Holes, International Communications in Heat and Mass Transfer 36(4), 342345, 2009.

Appendix Empirical results/Comparisons between dynamic and B-S model of average call price (Strike price 5600/5700/5900/6000) 
exercise price 5600
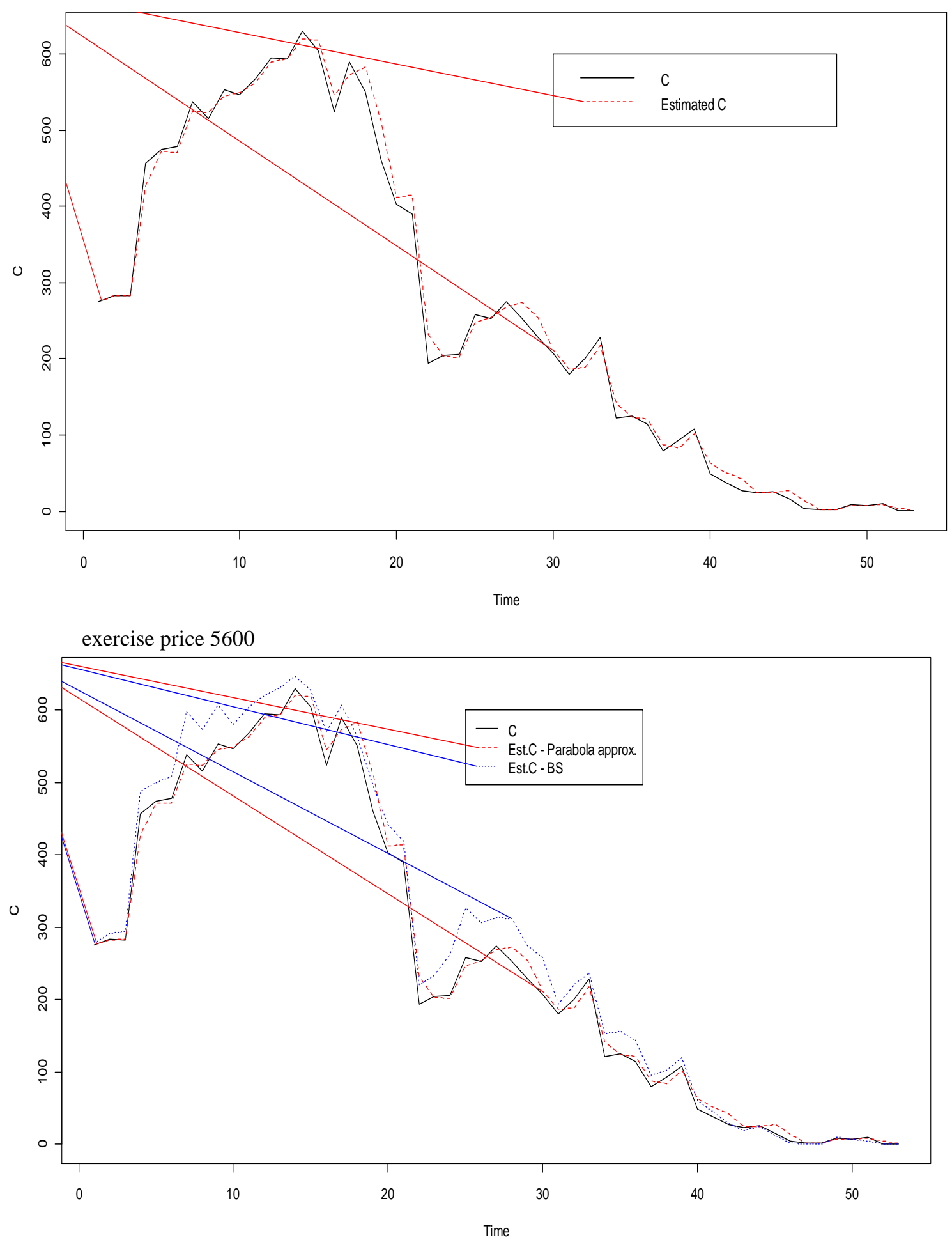
exercise price 5700
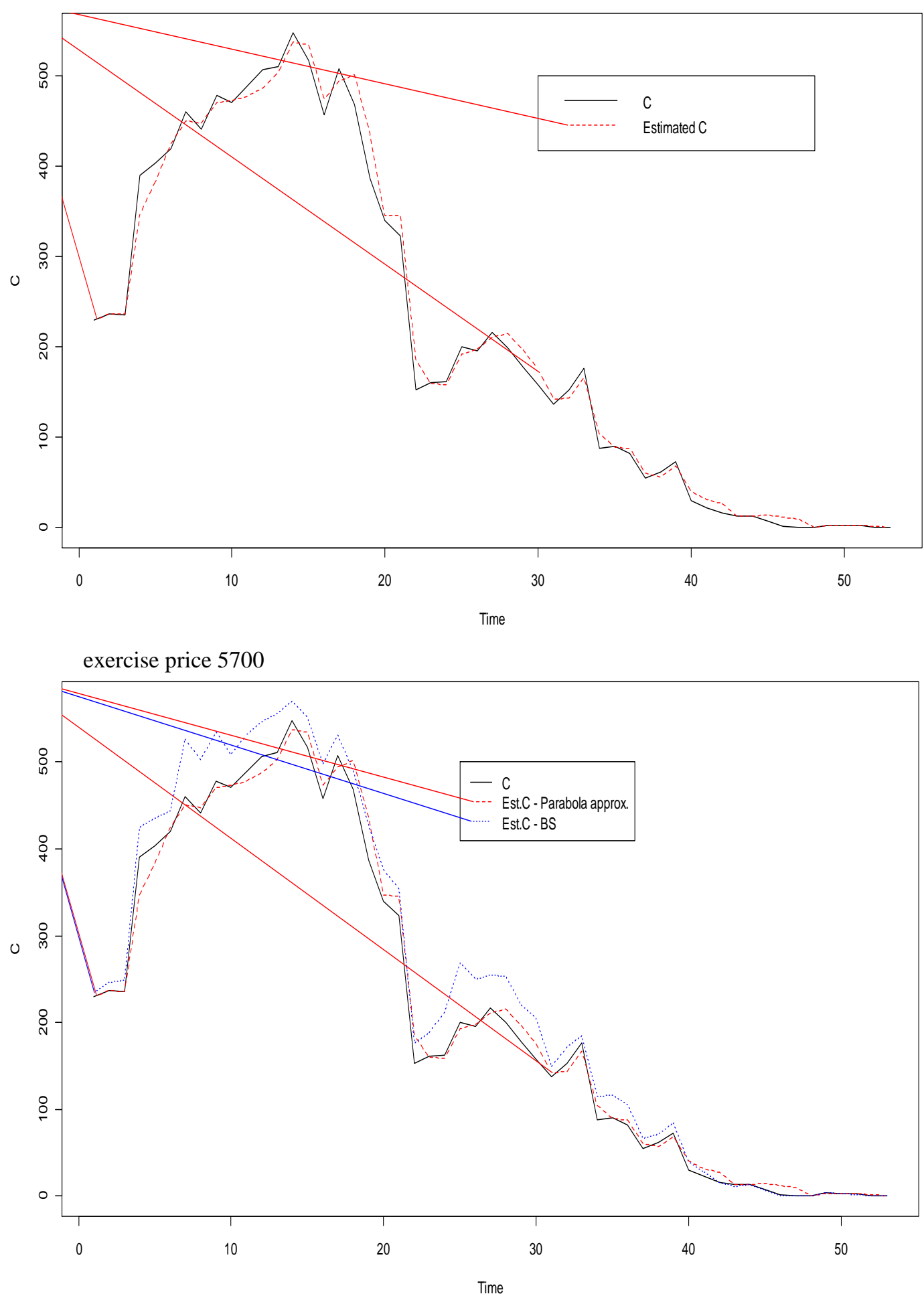
exercise price 5900

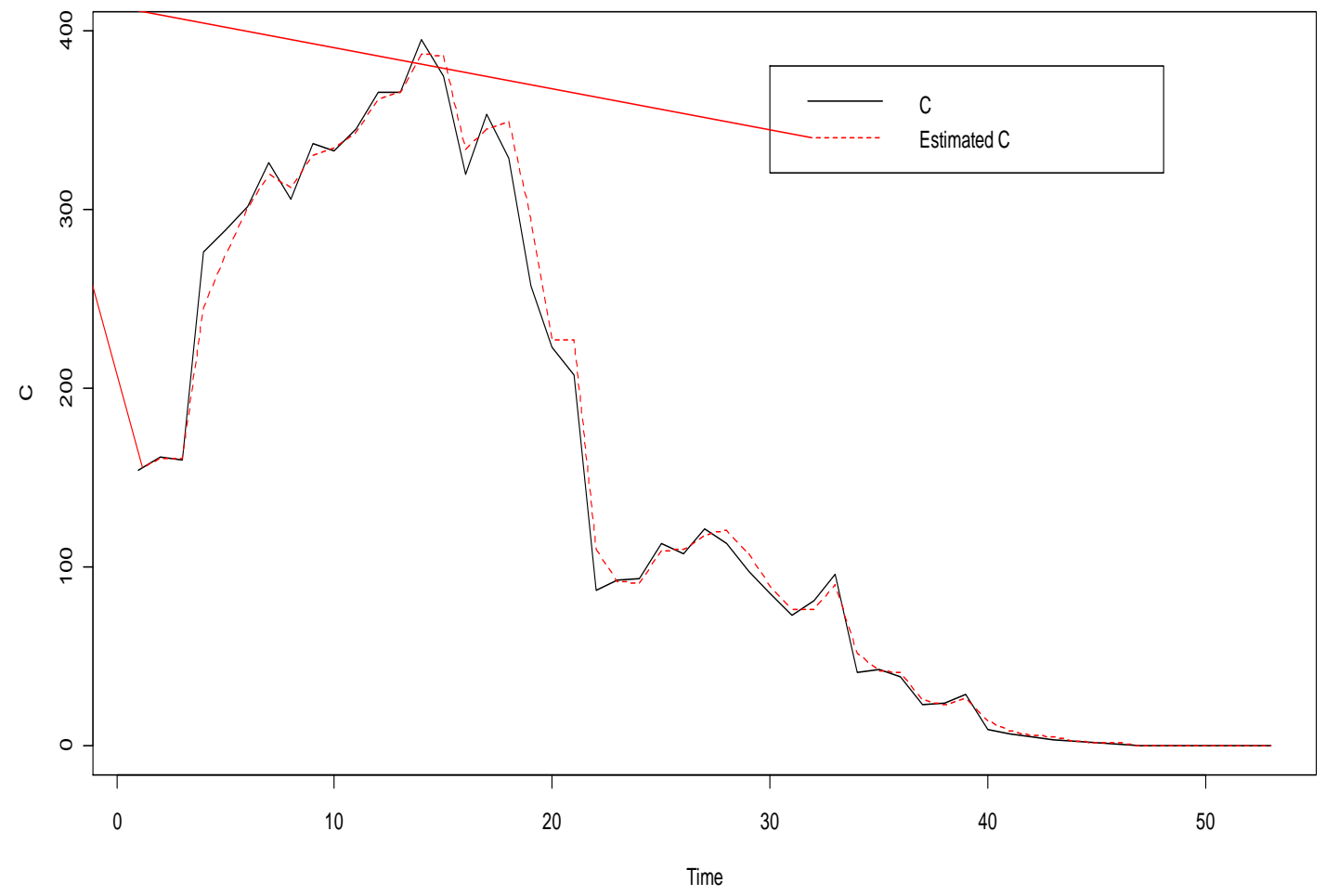

exercise price 5900

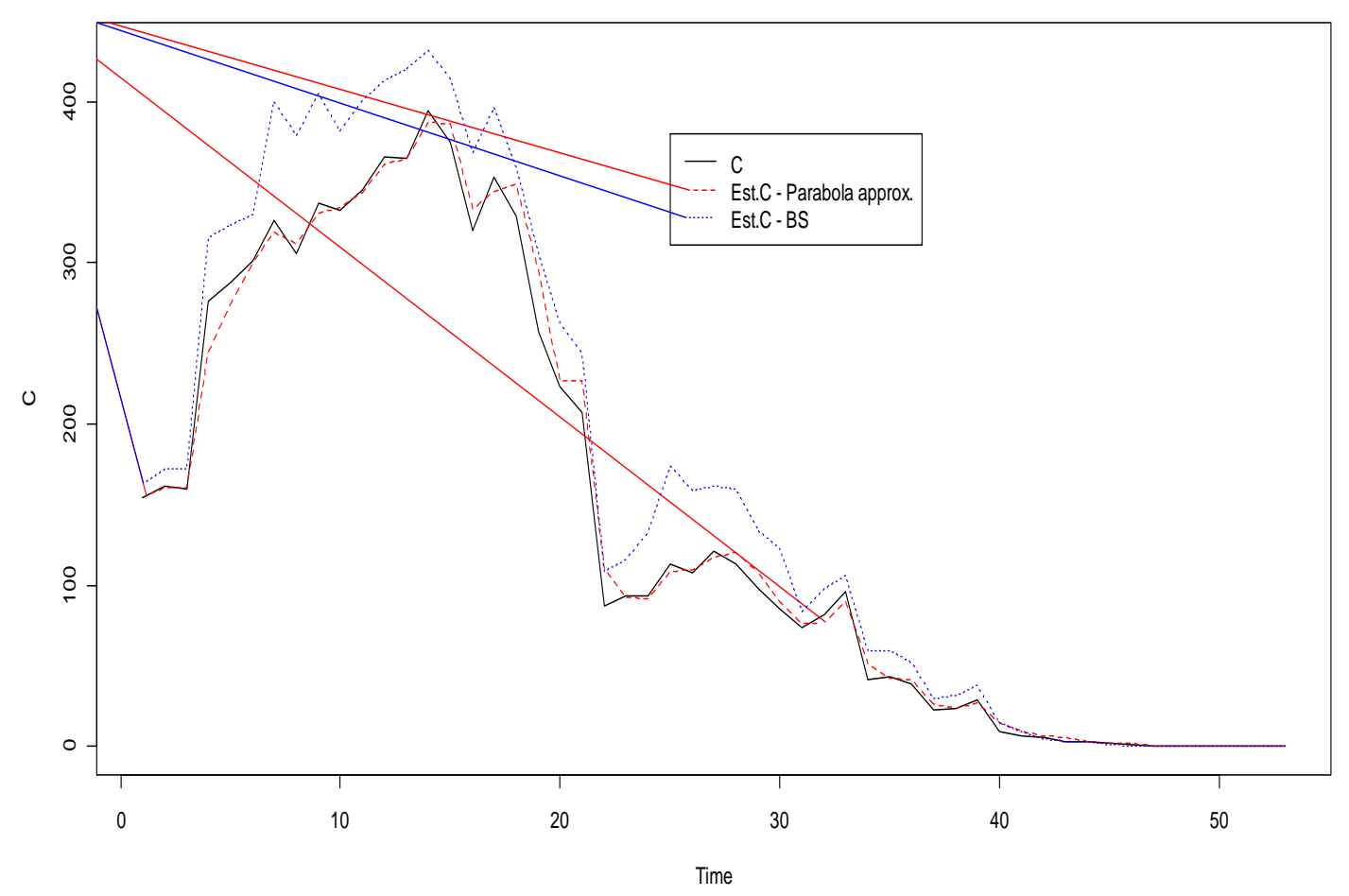


exercise price 6000

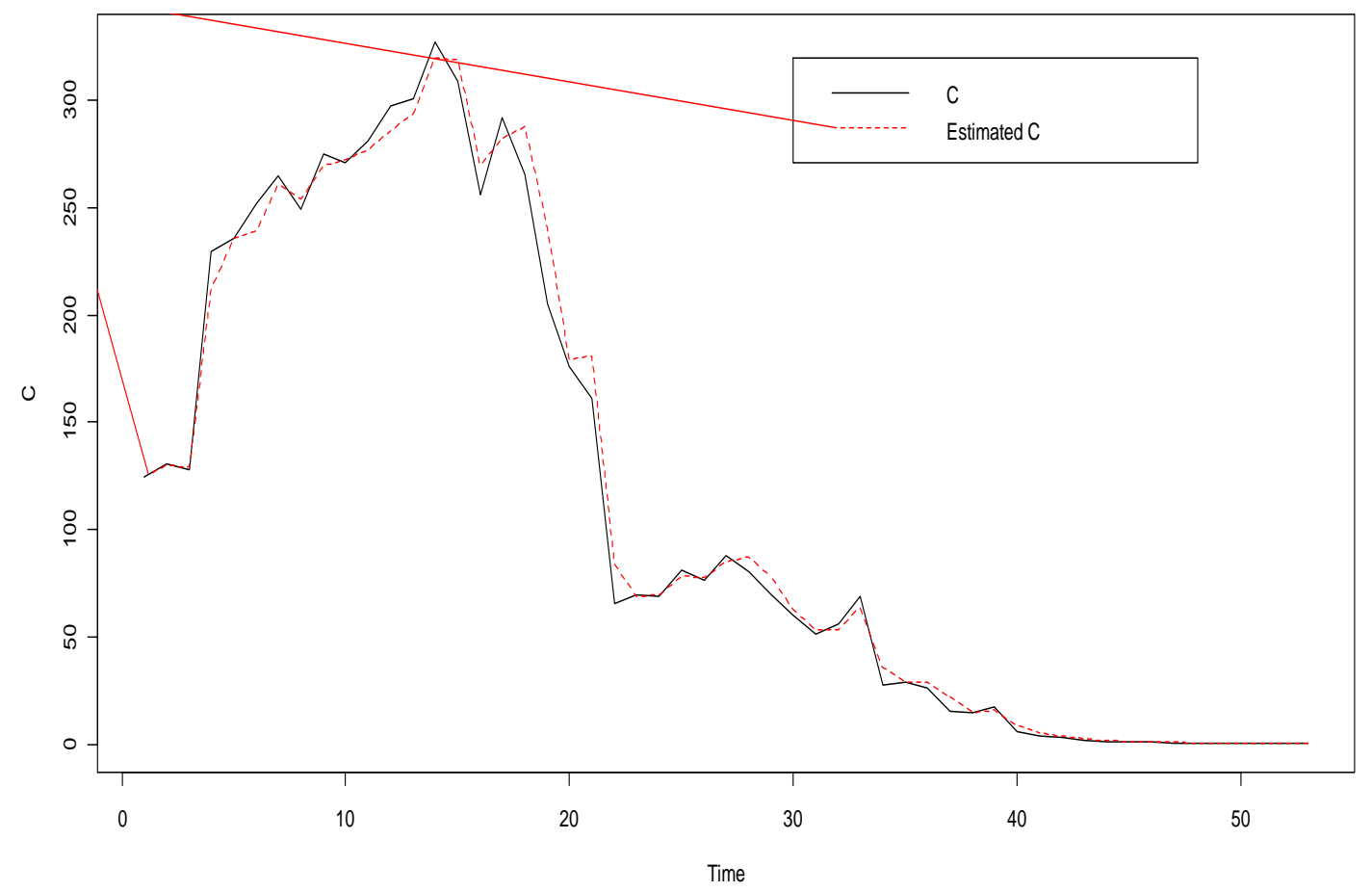

exercise price 6000

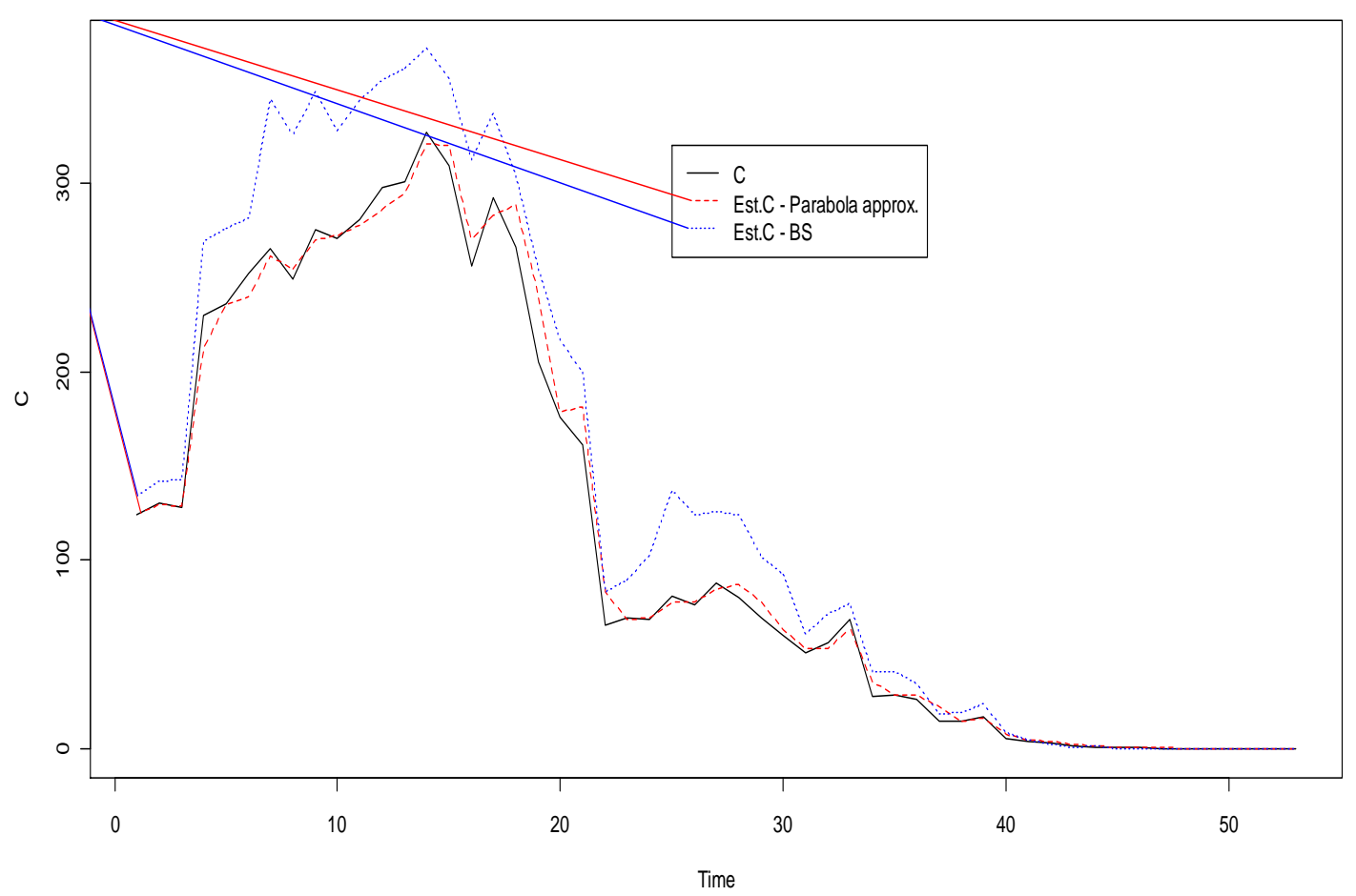

